\title{
COMPUTATION OF LENGTH SCALES FOR SECOND ORDER LAMINATED MICROSTRUCTURE WITH SURFACE ENERGY
}

\author{
LIYING LIU AND ZHIPING LI ${ }^{\dagger}$ \\ LMAM \& SCHOOL OF MATHEMATICAL SCIENCES, \\ PEKING UNIVERSITY, BEIJING 100871, P.R.CHINA
}

\begin{abstract}
A computational model for the total energy, including surface energy, of second order laminated microstructures is proposed. By studying the minimization property of this energy, we can numerically reveal how the twin widths of a second order laminated microstructure are related to the specimen's length and the surface energy density of a crystalline material. Numerical experiments on a 2-dimensional Ericksen-James type elastic model for crystals show that the twin widths of the first and second order laminates satisfy certain cubic rule.
\end{abstract}

\section{INTRODUCTION}

Microstructure is a commonly observed phenomenon in many elastic crystals. Martensite crystals are a kind of such crystals which have a high temperature phase (austenite) and a low temperature phase (martensite). Under certain temperature, austenite and martensite can transform from one to another, this temperature is called the transformation temperature. Austenite with a unique variant is a higher symmetric phase and martensite with several symmetry-related variants is a lower symmetric phase. Below the transformation temperature different variants of martensite can coexist and mix with each other in fine scales.

1991 Mathematics Subject Classification. 73C50,65K10,65N30.

Key words and phrases. energy minimization, second order laminated microstructure, length scales, surface energy density.

The research was supported in part by the Special Funds for Major State Basic Research Projects (2005CB321701), NSFC projects (10431050 and 10571006) and RFDP of China.

$\dagger$ Corresponding author: Zhiping Li, email address: lizp@math.pku.edu.cn. 
The simplest martensite microstructure is the so called simple laminates in which the twinned layers of two variants of martensite form into a laminate perpendicular to a certain direction. Laminates can be nested within a layer of another laminate to form a higher order laminated microstructure. In a laminated microstructure, a laminate is called the first order laminate if it is not nested in a layer of any other laminates, and is called a second order laminate if it is nested in and only in a layer of the first order laminate, and is called a $k$ th order laminate if it is nested exactly in a layer of a $(k-1)$ th order laminate. A laminated structure is called a $k$ th order laminated microstructure, if $k$ is the highest order of the laminates in the structure. Figure- 1 is an example of a higher order laminated microstructure [3].

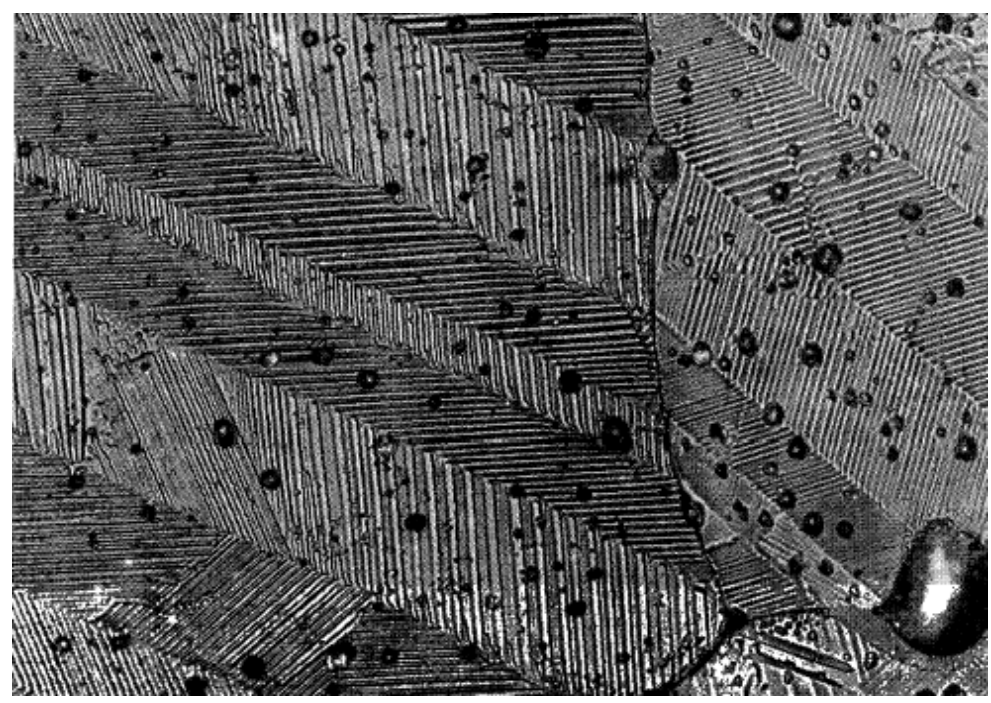

FiguRE 1. Higher order laminated microstructure

The length scales, that is the widths of the twinned layers of the laminates, are found to depend on the specimen's dimension as well as the surface energy density on the interfaces between the martensite variants [1, 8, 9]. Mathematical analysis and numerical computations on the length scales of simple laminated microstructures with surface energy can be found in $[5,8,9,16,17]$ among many others.

In the present paper, a mathematical model for computing the length scales of second order laminated structures is established. The idea is to minimize the total potential energy which is the sum of the elastic energy of the needle-like 
structures of the second order laminates near the interfaces between the layers of the first order laminate and the elastic energy near the specimen's boundary and the surface energy on the interfaces of second order laminates, and these potential energies can be considered as functions of the length scales, the specimen's dimension and the surface energy density defined on the sharp interfaces between the layers of the second order laminates. The key to an accurate numerical result is to accurately compute the elastic energy of the bending needle-like structures near the interfaces of the layers of the first order laminate. To minimize the mesh dependence of the numerical result, which can be significant and can cause serious problems $[11,17,19]$, the mesh transformation method is applied so that the mesh lines can follow the twin boundaries of the needle-like structures.

The rest of the paper is arranged as follows. In section 2, a mathematical model for the average energy density and the length scales of a second order laminated microstructure is established. The numerical experiments and results are presented in section 3, where we see that the twin widths of the first and second laminates satisfy certain cubic rule, more precisely, they are proportional to $\left(l^{2} e_{s}\right)^{1 / 3}$ and $\left(l e_{s}^{2}\right)^{1 / 3}$ respectively, where $l$ is the length of the square specimen and $e_{s}$ is the surface energy density defined on the interfaces between the two martensite variants.

\section{Average Energy Density and Length Scales of Second Order Laminated Microstructure}

\subsection{Structure of second order martensite laminated microstructure.} We restrict ourselves in the two-dimensional model. Let $\Phi(\nabla u(x), \theta)$ be the elastic energy density for deformation gradient $\nabla u$ at temperature $\theta$. Assume, for $\theta$ lower than the transformation temperature $\Theta_{T}$, the energy density $\Phi(\nabla u(x), \theta)$ has two energy wells $S O(2) U_{0}$ and $S O(2) U_{1}$, where $S O(2)=\left\{R \in R^{2 \times 2}: R^{T} R=\right.$ $I$, $\operatorname{det} R=1$.$\} is the set of all rotational matrixes, and$

$$
U_{0}=\left(\begin{array}{cc}
\sqrt{1-\hat{\varepsilon}} & 0 \\
0 & \sqrt{1+\hat{\varepsilon}}
\end{array}\right), \quad U_{1}=\left(\begin{array}{cc}
\sqrt{1+\hat{\varepsilon}} & 0 \\
0 & \sqrt{1-\hat{\varepsilon}}
\end{array}\right)
$$

where $\hat{\varepsilon}$ is the transformation strain. Then it is easily verified that there exist $R^{ \pm} \in S O(2)$ such that $U_{0}$ and $R^{ \pm} U_{1}$ are in rank-one connection. More precisely, 
let $\eta_{1}=\sqrt{1-\hat{\varepsilon}}, \eta_{2}=\sqrt{1+\hat{\varepsilon}}$, and let

$$
R^{ \pm}=\left(\begin{array}{ll}
\eta_{1} \eta_{2} & \pm \hat{\varepsilon} \\
\mp \hat{\varepsilon} & \eta_{1} \eta_{2}
\end{array}\right)
$$

then, we have

$$
R^{ \pm} U_{1}=U_{0}+\boldsymbol{a}^{ \pm} \otimes \boldsymbol{n}^{ \pm}
$$

where $\mathbf{a}^{ \pm}=\sqrt{2} \hat{\varepsilon}\left(\eta_{1}, \mp \eta_{2}\right)^{T}$ and $\mathbf{n}^{ \pm}=\frac{1}{\sqrt{2}}(1, \pm 1)^{T}$.

Thus the laminates can be formed between $U_{0}$ and $R^{ \pm} U_{1}$ with an arbitrary given volume fraction $\tau \in(0,1)$. We denote the laminates with the average deformation gradients of the form $Q^{+} A_{\mu}^{+} \triangleq Q^{+}\left((1-\mu) U_{0}+\mu R^{+} U_{1}\right)$ and $Q^{-} A_{\lambda}^{-} \triangleq$ $Q^{-}\left((1-\lambda) U_{0}+\lambda R^{-} U_{1}\right)$ for some $Q^{ \pm} \in S O(2)$ and $\mu, \lambda \in[0,1]$ the Martensite-1 and Martensite- 2 respectively. It is not difficult to show that the Martensite-1 and Martensite-2 are in rank-one connection. In fact, we have [12]

$$
A_{\mu}^{+}=Q A_{\lambda}^{-}+\boldsymbol{a} \otimes \boldsymbol{n}(\beta)
$$

where

$$
\begin{gathered}
Q=\left(\begin{array}{cc}
\cos \xi & -\sin \xi \\
\sin \xi & \cos \xi
\end{array}\right), \\
\xi=\arctan \frac{a \sqrt{a^{2}+b^{2}-\eta_{1}^{2} \eta_{2}^{2}}-b \eta_{1} \eta_{2}}{b \sqrt{a^{2}+b^{2}-\eta_{1}^{2} \eta_{2}^{2}}+a \eta_{1} \eta_{2}},
\end{gathered}
$$

where $a=\eta_{1} \eta_{2}\left(1-2 \lambda \mu \hat{\varepsilon}^{2}\right), b=(\lambda+\mu) \hat{\varepsilon}-2 \lambda \mu \hat{\varepsilon}^{3}$ and $\boldsymbol{n}(\beta)=(\cos \beta, \sin \beta)^{T}$.

For given $\lambda, \mu, \sigma \in[0,1]$ satisfying $(\lambda(1-\lambda)+\mu(1-\mu)) \sigma(1-\sigma)>0$, a second order laminated microstructure with average deformation gradient

$$
A_{\lambda \mu}^{\sigma}=(1-\sigma) A_{\mu}^{+}+\sigma Q A_{\lambda}^{-}
$$

can be constructed by the layers of Martensite-1 (with average deformation gradient $A_{\mu}^{+}$) and Martensite-2 (with average deformation gradient $Q A_{\lambda}^{-}$) with volume fractions $(1-\sigma)$ and $\sigma$ respectively, and it is easily seen, by $(2.4)$, that $\boldsymbol{n}(\beta)$ is the unit normal to the interfaces between the layers of the first order laminate (see Figure-2 and Figure-3). 


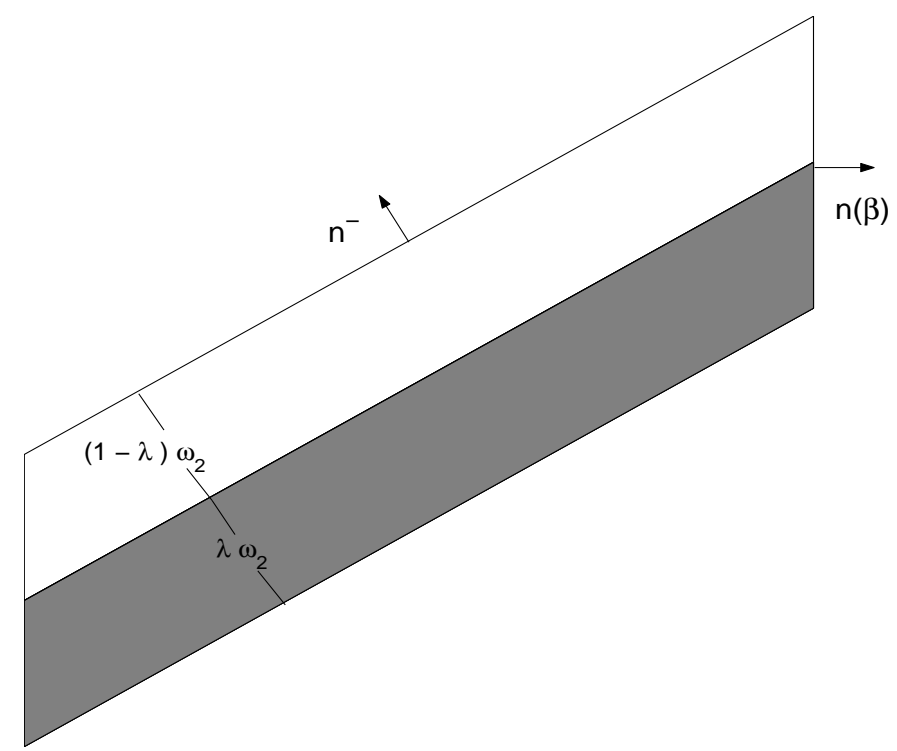

Figure 2. Twin layers of Martensite-2 laminate.

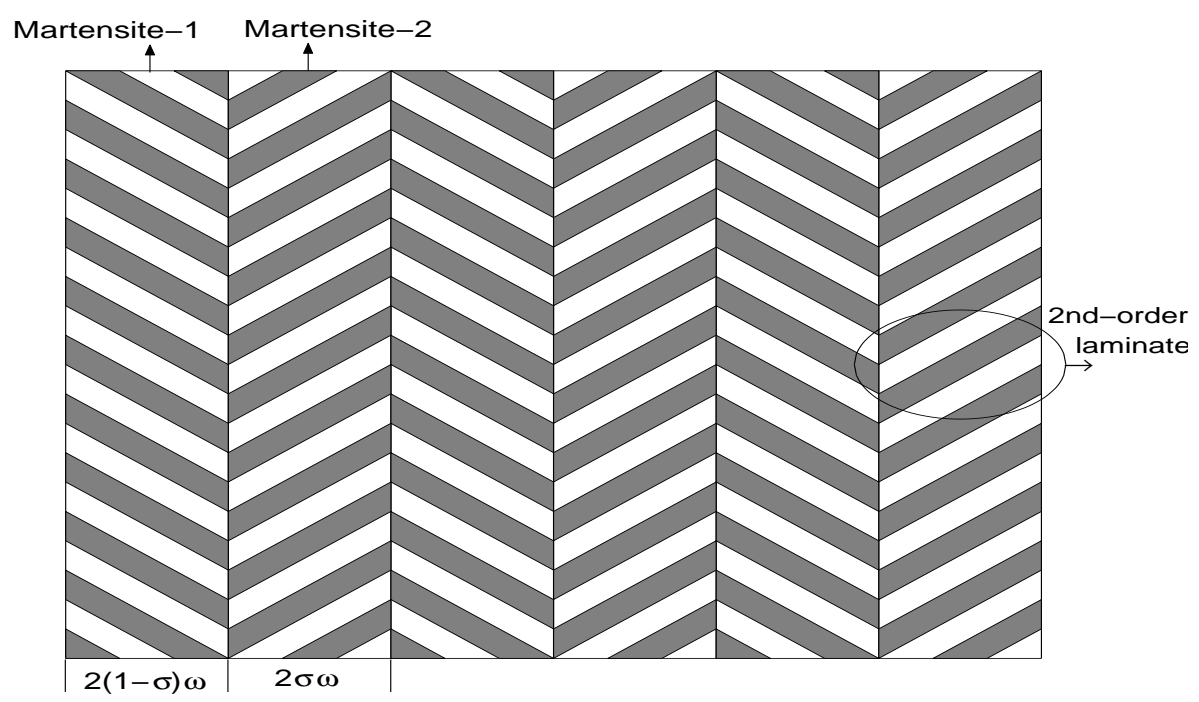

FIGURE 3. second order laminated microstructure constructed by Martensite-1 and Martensite-2 laminates.

2.2. Energy of needle-like structures. As the twinned layers of a second order laminate approach the interface of the first order laminate, they will, forced by the deformation continuity condition, bend into a needle-like structure satisfying certain energy minimizing property. 
In this subsection, taking Martensite-2 as an example (see [16] for further information on needle-like microstructures), we will discuss the energy of a needlelike structure.

By Figure 2 and (2.4) (see [16] for details), it is easily seen that, for $\alpha=-\pi / 4$ (notice that $\mathbf{n}^{-}=\mathbf{n}(-\pi / 4)$ ), the periodic relationship

$$
u\left(x+\frac{\omega_{2} \boldsymbol{n}\left(\beta+\frac{\pi}{2}\right)}{\left|\cos \left(\alpha-\beta+\frac{\pi}{2}\right)\right|}\right)-Q A_{\lambda}^{-}\left(x+\frac{\omega_{2} \boldsymbol{n}\left(\beta+\frac{\pi}{2}\right)}{\left|\cos \left(\alpha-\beta+\frac{\pi}{2}\right)\right|}\right)=u(x)-Q A_{\lambda}^{-} x
$$

holds for both the deformation of the twinned Martensite- 2 with the twin width $\omega_{2}$ and the average deformation of the twinned Martensite-1. This allows us to consider a periodic problem for the needle-like structure on a parallelogram with one pair of parallel sides, of which the length is $\left|\cos \left(\alpha-\beta+\frac{\pi}{2}\right)\right| \omega_{2}^{-1}$, perpendicular to $\boldsymbol{n}(\beta)$ and the other pair of parallel sides, of which the length is $l_{20}$, perpendicular to $\boldsymbol{n}(\alpha)$ (see Figure 2 and Figure 4 ). Let $\Omega_{2}$ be such a parallelogram centered at 0 . The boundary condition for the deformation $u(x)$ of the needle-like structure can be given by

$$
u_{n}(x)= \begin{cases}u_{n}\left(x \mp \frac{\omega_{2} \boldsymbol{n}\left(\beta+\frac{\pi}{2}\right)}{\left|\cos \left(\alpha-\beta+\frac{\pi}{2}\right)\right|}\right) \pm Q A_{\lambda}^{-} \frac{\omega_{2} \boldsymbol{n}\left(\beta+\frac{\pi}{2}\right)}{\left|\cos \left(\alpha-\beta+\frac{\pi}{2}\right)\right|}, & \text { on } \partial \Omega_{2}^{ \pm}, \\ Q U_{0}\left(x-\omega_{2}\left(\frac{1}{2}-\lambda\right) \boldsymbol{n}(\alpha)\right)+\int_{0}^{\omega_{2}^{-1} x \cdot \boldsymbol{n}(\alpha)} f_{\lambda}(s) \mathrm{ds} \boldsymbol{a}^{-}, & \text {on } \partial \Omega_{2+}, \\ A_{\mu}^{+}\left(x-\omega_{2}\left(\frac{1}{2}-\lambda\right) \boldsymbol{n}(\alpha)\right), & \text { on } \partial \Omega_{2-},\end{cases}
$$

where $\partial \Omega_{2}^{ \pm}$are the pair of sides perpendicular to $\boldsymbol{n}(\alpha)$ and $\partial \Omega_{2 \pm}$ are the pair of sides perpendicular to $\boldsymbol{n}(\beta)$, and

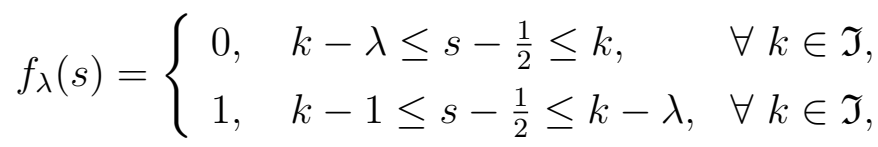

where $\mathfrak{I}$ is the set of all integers.

Notice that, if $u(x)=u_{n}(x)$ on $\partial \Omega_{2}$, then it satisfies the periodic relation (2.8) and takes the value of the Martensite-2 twin laminate on $\partial \Omega_{2+}$ and a linear function, which reflects the average deformation gradient of the Martensite- 2 as well as that of the Martensite- 1 twin laminates, on $\partial \Omega_{2-}$. 


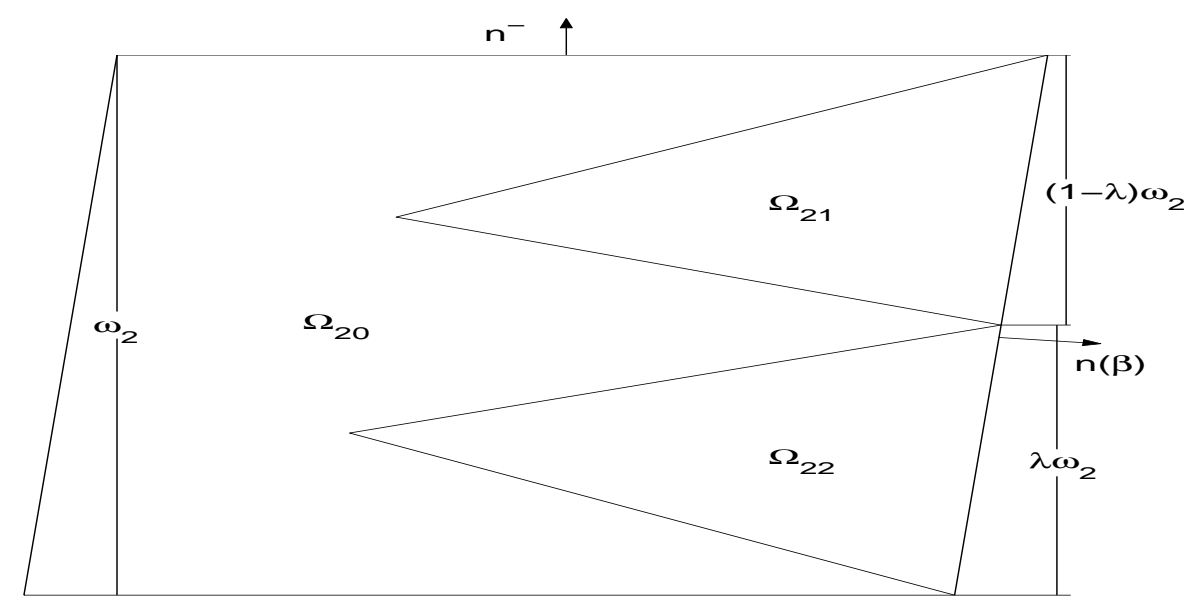

Figure 4. Admissible decomposition of $\Omega_{2}$ for needles

The domain $\Omega_{2}$ is said to have an admissible decomposition for needles, if $\Omega_{2}$ can be divided into three disjoint connected subdomains $\Omega_{2 i}, i \in\{0,1,2\}$, with Lipschitz continuous boundaries $\partial \Omega_{2}^{i}=\partial \Omega_{2 i} \backslash \partial \Omega_{2 \pm}, i=1$, 2, each of which consists of two piecewise smooth curves as shown in Figure 4.

Denote

$$
U\left(u_{n} ; \Omega_{2}\right)=\left\{u \in W^{1, p}:\left.u\right|_{\partial \Omega_{2}}=u_{n}\right\},
$$

where $p>2$ is determined by the energy density $\Phi(\cdot, \theta)$. Let the set of admissible functions $U_{n}\left(u_{n} ; \Omega_{2}\right) \subset U\left(u_{n} ; \Omega_{2}\right)$ be the set of all functions $u \in U\left(u_{n} ; \Omega_{2}\right)$ such that the sets

$$
\begin{aligned}
& \Omega_{20}=\left\{x \in \Omega_{2}:\left|\nabla u(x)-A_{\mu}^{+}\right|<\left|\nabla u(x)-Q R^{-} U_{1}\right| \wedge\left|\nabla u(x)-Q U_{0}\right|\right\}, \\
& \Omega_{21}=\left\{x \in \Omega_{2}: \nabla u(x)-Q U_{0}|\leq| \nabla u(x)-Q R^{-} U_{1}|\wedge| \nabla u(x)-A_{\mu}^{+} \mid\right\}, \\
& \Omega_{22}=\left\{x \in \Omega_{2}:\left|\nabla u(x)-Q R^{-} U_{1}\right| \leq\left|\nabla u(x)-A_{\mu}^{+}\right| \wedge\left|\nabla u(x)-Q U_{0}\right|\right\},
\end{aligned}
$$

where $\alpha \wedge \beta=\min \{\alpha, \beta\}$, give $\Omega_{2}$ an admissible decomposition for needles.

Let $v \in U_{n}\left(u_{n} ; \Omega_{2}\right)$, denote the elastic energy of the corresponding twin needles by

$$
\tilde{E}_{2 n}\left(l_{20}, \omega_{2}, v\right) \triangleq F\left(v ; \Omega_{2}\right)=\int_{\Omega_{2}} \Phi(\nabla v, \theta) d x,
$$

and define the elastic energy of the minimizing twin needles by

$$
E_{2 n}\left(l_{20}, \omega_{2}\right)=\inf _{v \in U_{n}\left(u_{n} ; \Omega_{2}\right)} \tilde{E}_{2 n}\left(l_{20}, \omega_{2}, v\right) .
$$


To calculate $E_{2 n}\left(l_{20}, \omega_{2}\right)$ is equivalent to solve the problem

$$
\left\{\begin{array}{l}
\text { find } u \in U_{n}\left(u_{n} ; \Omega_{2}\right) \text { such that } \\
F\left(u ; \Omega_{2}\right)=\inf _{v \in U_{n}\left(u_{n} ; \Omega_{2}\right)} F\left(v ; \Omega_{2}\right) .
\end{array}\right.
$$

Let $v \in U_{n}\left(u_{n} ; \Omega_{2}\right)$, denote by $\tilde{l}_{2 n}\left(l_{20}, \omega_{2}, v\right)$ the total length of the corresponding twin needles' interface $\partial \Omega_{2}^{1} \cup \partial \Omega_{2}^{2}$. Define

$$
l_{2 n}\left(l_{20}, \omega_{2}\right)=\inf _{\left\{u^{(\alpha)}\right\}} \liminf _{\alpha \rightarrow \infty} \tilde{l}_{2 n}\left(l_{20}, \omega_{2}, u^{(\alpha)}\right),
$$

where the infimum is taken among all the possible minimizing sequences $\left\{u^{(\alpha)}\right\} \subset$ $U_{n}\left(u_{n} ; \Omega_{2}\right)$ of the problem (2.11). By a change of the integral variables, we have the following scaling relations [16]

$$
E_{2 n}\left(\delta l_{20}, \delta \omega_{2}\right)=\delta^{2} E_{2 n}\left(l_{20}, \omega_{2}\right)
$$

and

$$
l_{2 n}\left(\delta l_{20}, \delta \omega_{2}\right)=\delta l_{2 n}\left(l_{20}, \omega_{2}\right) .
$$

For given surface energy density $e_{s}$ and the size of $\Omega_{2}: l_{20}>\omega_{2}>0$, the total potential energy of the twin needles is given as the sum of the elastic energy $E_{2 n}\left(l_{20}, \omega_{2}\right)$ and the surface energy $e_{s} l_{2 n}\left(l_{20}, \omega_{2}\right)$. Similar results can also be obtained for the twin needles of Martensite-1.

2.3. Total energy of Martensite-1 and Martensite-2 laminates. The total energy of the Martensite-1 and Martensite-2 laminates includes four parts: the elastic energy of the twin laminates, the elastic energy of the twin needles, the surface energy on the interfaces of the twin laminates and the surface energy for the twin needles. More precisely, taking the Martensite-2 laminate with the total length $l_{2}$ and needle length $l_{20}$ and twin width $\omega_{2}$ as an example, we can write the total energy on a twinned layers of the Martensite-2 laminate as [16]

$$
\tilde{E}_{2}\left(l_{2}, l_{20}, \omega_{2}\right)=\Phi_{\theta}\left(l_{2}-l_{20}\right) \omega_{2}+E_{2 n}\left(l_{20}, \omega_{2}\right)+2\left(l_{2}-l_{20}\right) e_{s}+l_{2 n}\left(l_{20}, \omega_{2}\right) e_{s},
$$

where $e_{s}$ is the surface energy density and

$$
\Phi_{\theta}=\Phi\left(U_{0}, \theta\right)=\Phi\left(U_{1}, \theta\right)=\min _{\left\{U \in R^{2 \times 2}: \operatorname{det} U>0\right\}} \Phi(U, \theta)
$$

is the elastic energy density of the martensite variants at temperature $\theta$. Since on a unit length in $\mathbf{n}(\alpha)=\mathbf{n}^{-}$direction there are $\omega^{-1}$ pairs of the twin layers, 
by (2.15) and the scaling relations (2.13) and (2.14), the total energy of the corresponding Martensite-2 laminate on per unit width in $\boldsymbol{n}(\alpha)$ direction can be written as

$$
\begin{array}{r}
\hat{E}_{2}\left(l_{2}, \hat{l}_{20}, \omega_{2}\right) \triangleq \tilde{E}_{2}\left(l_{2}, l_{20}, \omega_{2}\right) \omega_{2}^{-1}=\Phi_{\theta}\left(l_{2}-\hat{l}_{20} \omega_{2}\right)+E_{2 n}\left(\hat{l}_{20}, 1\right) \omega_{2}+ \\
2 l_{2} e_{s} \omega_{2}^{-1}+\left(l_{2 n}\left(\hat{l}_{20}, 1\right)-2 \hat{l}_{20}\right) e_{s}
\end{array}
$$

where $\hat{l}_{20}=l_{20} \omega_{2}^{-1}$ is the so called the characteristic length of the needles.

By the energy minimization principle, for a given specimen of Martensite-2 laminate with total laminate length $l_{2}$, the twin width $\omega_{2}$ and the minimizing characteristic needle length $\hat{l}_{20}$ are given by the solution of the following problem

$$
\hat{E}_{2}\left(l_{2}, \hat{l}_{20}, \omega_{2}\right)=\inf _{\tilde{l}_{20} \in\left[\tau, l_{2} \tilde{\omega}^{-1}\right], \tilde{\omega}>0} \hat{E}_{2}\left(l_{2}, \tilde{l}_{20}, \tilde{\omega}\right),
$$

where $\tau>0$ is a constant depend loosely on the elastic properties of the crystal.

Theorem 2.1. Assume that $\left(\hat{l}_{20}, \omega_{2}\right)$ is a solution of (2.17), then

$$
\omega_{2}=\hat{\omega}_{2}\left(\hat{l}_{20}\right) \triangleq\left(\frac{2 l_{2} e_{s}}{E_{2 n}\left(\hat{l}_{20}, 1\right)-\Phi_{\theta} \hat{l}_{20}}\right)^{\frac{1}{2}}
$$

and thus the minimizing energy of Martensite-2 laminate has the form

$$
\begin{aligned}
E_{2}\left(l_{2}, \hat{l}_{20}\right) & \triangleq \hat{E}_{2}\left(l_{2}, \hat{l}_{20},\left(\frac{2 l_{2} e_{s}}{E_{2 n}\left(\hat{l}_{20}, 1\right)-\Phi_{\theta} \hat{l}_{20}}\right)^{\frac{1}{2}}\right) \\
& =\Phi_{\theta} l_{2}+2\left(2 l_{2} e_{s}\left(E_{2 n}\left(\hat{l}_{20}, 1\right)-\Phi_{\theta} \hat{l}_{20}\right)\right)^{\frac{1}{2}}+\left(l_{2 n}\left(\hat{l}_{20}, 1\right)-2 \hat{l}_{20}\right) e_{s}
\end{aligned}
$$

Proof. Since $\left(\hat{l}_{20}, \omega_{2}\right)$ minimizes the energy $\hat{E}_{2}\left(l_{2}, \hat{l}_{20}, \omega_{2}\right)$, we have, by $(2.16)$,

$$
\frac{\partial \hat{E}_{2}\left(l_{2}, \hat{l}_{20}, \omega_{2}\right)}{\partial \omega_{2}}=-\Phi_{\theta} \hat{l}_{20}+E_{2 n}\left(\hat{l}_{20}, 1\right)-2 l_{2} e_{s} \omega_{2}^{-2}=0
$$

and thus the theorem follows.

Similarly, for Martensite-1 laminate, we have

$$
\hat{E}_{1}\left(l_{1}, \hat{l}_{10}, \omega_{1}\right)=\inf _{\tilde{l}_{10} \in\left[\tau, l_{1} \tilde{\omega}^{-1}\right], \tilde{\omega}>0} \hat{E}_{1}\left(l_{1}, \tilde{l}_{10}, \tilde{\omega}\right),
$$


and

$$
\omega_{1}=\hat{\omega}_{1}\left(\hat{l}_{10}\right) \triangleq\left(\frac{2 l_{1} e_{s}}{E_{1 n}\left(\hat{l}_{10}, 1\right)-\Phi_{\theta} \hat{l}_{10}}\right)^{\frac{1}{2}} .
$$

For sufficiently small surface energy density $e_{s}$, if $\hat{l}_{i 0}, i=1$ (or 2 ), is a solution to the following minimization problem (see [16])

$$
\begin{aligned}
& E_{i}\left(l_{i}, \hat{l}_{i 0}\right)=\inf _{\tilde{l}_{i 0}>\tau} E_{i}\left(l_{i}, \tilde{l}_{i 0}\right) \\
& =\inf _{\tilde{l}_{i 0}>\tau}\left[\Phi_{\theta} l_{i}+2\left(2 l_{i} e_{s}\left(E_{i n}\left(\tilde{l}_{i 0}, 1\right)-\Phi_{\theta} \tilde{l}_{i 0}\right)\right)^{\frac{1}{2}}+\left(l_{i n}\left(\tilde{l}_{i 0}, 1\right)-\tilde{l}_{i 0}\right) e_{s}\right]
\end{aligned}
$$

and $\hat{\omega}_{i}\left(\hat{l}_{i 0}\right)$ is given by $(2.21)$ (or $\left.(2.18)\right)$, then $\left(\hat{l}_{i 0}, \hat{\omega}_{i}\left(\hat{l}_{i 0}\right)\right)$ solves $(2.20)$ (or $\left.(2.17)\right)$.

2.4. Elastic energy in the boundary layer. The energy on the specimen's boundary is also an important part of the total potential energy in determining the length scales of the second order laminated microstructure. In our model, the hard boundary condition, i.e. the Dirichlet boundary condition, is used, thus the energy on the specimen's boundary is given by the elastic energy defined on a boundary layer of the specimen. Since the first order laminate is periodic in the direction $\boldsymbol{n}(\beta)$, for simplicity, we consider a rectangular specimen with its sides either parallel or perpendicular to $\boldsymbol{n}(\beta)$ and the length in $\boldsymbol{n}(\beta)$ direction being a multiple of the period (i.e. the twin width $2 \omega$ ) (see Figure 3). Thus we only need to compute the boundary layer elastic energy for a twinned layers of the first order laminate on the part of the specimen's boundary which is parallel to $\boldsymbol{n}(\beta)$.

Taking a layer of Martensite-2 as an example, we show how the boundary layer elastic energy is computed. Let $\Omega_{2 b}$ be a rectangle centered at 0 with one pair of sides $\Omega_{2 b}^{ \pm}$, of which the length is $2 \sigma \omega$, parallelling to $\boldsymbol{n}(\beta)$ and the length of the other pair of sides $\Omega_{2 b \pm}$ is $\nu$ (the width of the boundary layer). Let the boundary condition of $u(x)$ be given by

$$
u_{b}(x)= \begin{cases}Q A_{\lambda}^{-} x, & x \in \partial \Omega_{2 b \pm}, \\ A_{\lambda \mu} x, & x \in \partial \Omega_{2 b}^{+}, \\ Q A_{\lambda}^{-} x, & x \in \partial \Omega_{2 b}^{-} .\end{cases}
$$


Notice that on $\Omega_{2 b \pm}$ the macroscopic deformation of Martensite- 2 is imposed. This approximation is reasonable and necessary in computation, since $\omega_{2}<<\omega$. Now, the corresponding boundary layer elastic energy $\tilde{E}_{2 b}(2 \sigma \omega, \nu)$ can be obtained by solving the following problem

$$
\left\{\begin{array}{l}
\text { find } u \in U\left(u_{b} ; \Omega_{2 b}\right) \text { such that } \\
F\left(u ; \Omega_{2 b}\right)=\inf _{v \in U\left(u_{b} ; \Omega_{2 b}\right)} F\left(v ; \Omega_{2 b}\right) .
\end{array}\right.
$$

The boundary layer elastic energy $\tilde{E}_{1 b}(2(1-\sigma) \omega, \nu)$ corresponding to Martensite-1 can be obtained in the same way.

The average boundary layer elastic energy of Martensite- 1 and Martensite-2 in the boundary layer with length $\omega$ (in the $\boldsymbol{n}(\beta)$ direction) and width $\nu$ is given by

$$
\tilde{E}_{b}(\omega, \nu)=\frac{1}{2}\left[\tilde{E}_{1 b}(2(1-\sigma) \omega, \nu)+\tilde{E}_{2 b}(2 \sigma \omega, \nu)\right]
$$

By a change of the integral variables, it is easily seen that the scaling relation

$$
\tilde{E}_{b}(\omega, \nu)=\omega^{2} \tilde{E}_{b}\left(1, \omega^{-1} \nu\right)
$$

holds for all $\omega>0$ and $\nu>0$.

2.5. Energy of second order laminated microstructure. For a given square specimen of a second order laminated microstructure with its sides of length $l$ either parallel or orthogonal to $\mathbf{n}(\beta)$, the total potential energy is the sum of the total energy of Martensite- 1 and Martensite-2 laminates and the elastic energy in the boundary layer, that is

$$
\begin{array}{r}
E_{T}(l, \omega)=(l-2 \nu)\left[2\left(E_{1}\left(l_{1}(\omega), \hat{l}_{10}(\omega)\right)+E_{2}\left(l_{2}(\omega), \hat{l}_{20}(\omega)\right)\right)\right] \cdot \frac{l}{2 \omega}+ \\
2 \tilde{E}_{b}(\omega, \nu) \cdot \frac{l}{\omega}
\end{array}
$$

where $2 \omega$ is the twin width of the first order laminate, and

$$
l_{1}(\omega)=(1-\sigma) \omega\left|\cos \left(\beta-\frac{\pi}{2}-\alpha_{1}\right)\right|^{-1}, \quad l_{2}(\omega)=\sigma \omega\left|\cos \left(\beta+\frac{\pi}{2}-\alpha_{2}\right)\right|^{-1},
$$

with $\alpha_{1}=\pi / 4$ and $\alpha_{2}=-\pi / 4$ (recalling $\mathbf{n}^{ \pm}=\mathbf{n}( \pm \pi / 4)$ ), and $\nu$ is the width of the boundary layer, and where $\hat{l}_{10}(\omega)$ and $\hat{l}_{20}(\omega)$ are the minimizing characteristic needle lengths of Martensite-1 and Martensite-2 needles (see (2.17) and (2.22)). 
Hence, by (2.26) and the scaling relation (2.25), the average energy density of the corresponding second order laminated microstructure is given by

$$
\begin{aligned}
\bar{E}_{T}(l, \omega, \hat{\nu})=\left[E_{1}\left(l_{1}(\omega), \hat{l}_{10}(\omega)\right)+E_{2}\left(l_{2}(\omega), \hat{l}_{20}(\omega)\right)\right] \frac{1}{\omega}+ \\
2\left[\tilde{E}_{b}(1, \hat{\nu}) \omega-\left(E_{1}\left(l_{1}(\omega), \hat{l}_{10}(\omega)\right)+E_{2}\left(l_{2}(\omega), \hat{l}_{20}(\omega)\right)\right) \hat{\nu}\right] \frac{1}{l},
\end{aligned}
$$

where $\hat{\nu} \triangleq \nu \omega^{-1}$ is the characteristic boundary layer width. Define

$$
E_{b}(\omega, \hat{\nu})=\tilde{E}_{b}(1, \hat{\nu}) \omega-\left(E_{1}\left(l_{1}(\omega), \hat{l}_{10}(\omega)\right)+E_{2}\left(l_{2}(\omega), \hat{l}_{20}(\omega)\right)\right) \hat{\nu} .
$$

Denote $\hat{v}(\omega)$ the minimizer of the following problem

$$
E_{b}(\omega, \hat{\nu}(\omega))=\inf _{\tilde{\nu}>0} E_{b}(\omega, \tilde{\nu}) .
$$

Now, for given specimen's length $l$ and the surface energy density $e_{s}$, the average energy density of a second order laminated microstructure $\bar{E}_{T}(l, \omega, \hat{\nu}(\omega))$ can be viewed as a function of the first order laminate's twin width $\omega$. Thus, the energy minimizing principle leads to the consideration of the problem

$$
\left\{\begin{array}{l}
\text { Find } \omega>0 \text { such that } \\
\bar{E}_{T}(l, \omega, \hat{\nu}(\omega))=\inf _{\tilde{\omega}>0} \bar{E}_{T}(l, \tilde{\omega}, \hat{\nu}(\tilde{\omega})) .
\end{array}\right.
$$

2.6. Length scales of second order laminated microstructure. The length scales of the second order laminated microstructure, that is the twin width $\omega$ of the first order laminate, the twin widths $\omega_{1}$ and $\omega_{2}$ of the second order twin laminates of Martensite- 1 and Martensite-2, should be taken so that the average energy density $\bar{E}_{T}(l, \omega, \hat{\nu}(\omega))$ is minimized. Hence, to study the scaling law, we need to recover the the average energy density $\bar{E}_{T}(l, \omega, \hat{\nu}(\omega))$ as a function of the specimen's dimension $l$, the surface energy density $e_{s}$ and the twin width $\omega$. For given $\left(l, e_{s}, \omega\right)$ with $e_{s}$ sufficiently small, by (2.28), the function can be evaluated by solving the problems $(2.22)$ and $(2.29)$ using the functions $E_{i n}\left(\tilde{l}_{i 0}, 1\right), l_{i n}\left(\tilde{l}_{i 0}, 1\right)$ and $\tilde{E}_{i b}(1, \hat{\nu})(i=1,2)$ obtained by solving the corresponding minimization problems for needle structures and boundary layers (see (2.11) and (2.23)).

In computation, the functions $E_{i n}\left(\tilde{l}_{i 0}, 1\right), l_{i n}\left(\tilde{l}_{i 0}, 1\right)$ and $\tilde{E}_{i b}(1, \hat{\nu})(i=1,2)$ are approximated by data fitting using numerically obtained discrete sample data 
$\left\{E_{i n}\left(\tilde{l}_{i 0}^{(j)}, 1\right)\right\}_{j=1}^{J},\left\{l_{i n}\left(\tilde{l}_{i 0}^{(j)}, 1\right)\right\}_{j=1}^{J}$ and $\left\{\tilde{E}_{i b}\left(1, \hat{\nu}^{k}\right)\right\}_{k=1}^{K}$. Substitute these approximate functions into (2.22) and (2.28), the function $\bar{E}_{T}(l, \omega, \hat{\nu}(\omega))$ can be evaluated approximately, and the length scales can then be obtained by solving the problem (2.30) numerically using, for instance, the golden section method.

\section{Numerical Experiments And Results}

In our numerical experiments the following Ericksen-James type two dimensional energy density $\Phi(\nabla u(x), \theta) \triangleq \Phi(C, \theta)$ for elastic crystals is considered:

$$
\begin{aligned}
\Phi(C, \theta)=\frac{b(\theta)}{4}\left(C_{11}\right. & \left.-C_{22}\right)^{2}-\frac{c(\theta)}{8}\left(C_{11}-C_{22}\right)^{2}\left|C_{11}-C_{22}\right| \\
& +\frac{d(\theta)}{16}\left(C_{11}-C_{22}\right)^{4}+f C_{12}^{2}+g(t r C-2)^{2},
\end{aligned}
$$

where

$$
\begin{array}{r}
C \triangleq\left(C_{i j}\right)=(\nabla u)^{T} \nabla u, \quad b(\theta)=\left[1+\alpha \arctan \xi\left(\theta-\theta_{T}\right)\right] d_{0} \hat{\varepsilon}^{2}, \\
c(\theta)=2\left[1+\frac{1+2 \gamma}{3} \alpha \arctan \xi\left(\theta-\theta_{T}\right)\right] d_{0} \hat{\varepsilon}, \quad d(\theta)=\left[1+\gamma \alpha \arctan \xi\left(\theta-\theta_{T}\right)\right] d_{0},
\end{array}
$$

and where $d_{0}>0, f>0, g>0$ are the elastic moduli, $\hat{\varepsilon}$ is the transformation strain, $\alpha \approx \frac{2}{\pi}, \xi>0, \gamma<1$ are the material constants used to reflect the change of elastic moduli and the energy barriers as the temperature varies. It is known [16] that, for $\theta<\theta_{T}, \Phi(C, \theta)$ has two symmetry related rotationally invariant potential wells $S O(2) U_{0}$ and $S O(2) U_{1}$ defined by (2.1). In our numerical experiments, we set $\alpha=\frac{2.02}{\pi}, \xi=0.25, \gamma=0, \hat{\varepsilon}=0.05, d_{0}=500, f=3.5, g=15$, $\theta=-20{ }^{\circ} \mathrm{C}, \theta_{T}=70^{\circ} \mathrm{C}$.

For simplicity, in our computation, we take $\lambda=0.5, \mu=1$ and $\sigma=0.5$, thus there is only one martensite variant (no laminated structure) in Martensite-1 and we only need to consider Martensite-2.

Let $\Omega_{2}$ be a parallelogram as shown in Figure 4 with $\omega_{2}=0.1$ and the sample characteristic length $\hat{l}_{20}$ varies from 7.5 to 25 . Let the initial mesh be a uniformly distributed "English flag" shaped mesh introduced by $N \times M$ lines parallel to the sides and $(N+M) / 2$ lines parallel to each of the diagonals. The initial mesh is introduced by taking $\mathrm{M}=\mathrm{N}=8$, and is refined by a factor of 2 whenever it is necessary. Let $\Omega_{20}, \Omega_{21}$ and $\Omega_{22}$ be a decomposition of $\Omega_{2}$ as shown in Figure 4 
with the area of both $\Omega_{21}$ and $\Omega_{22}$ slightly greater than one eighth of the area of $\Omega_{2}$, and let the initial deformation $u_{0}(x)$ be given in the following way

$$
u_{0}\left(x^{j}\right)= \begin{cases}Q U_{0} x^{j}, & \text { if } x^{j} \in \mathfrak{N} \text { and } x^{j} \in \bar{\Omega}_{21}, \\ Q R^{-} U_{1} x^{j}, & \text { if } x^{j} \in \mathfrak{N} \text { and } x^{j} \in \bar{\Omega}_{22}, \\ R^{+} U_{1} x^{j}, & \text { if } x^{j} \in \partial \Omega_{2-}, \\ u_{n}\left(\Omega_{2-}, \Omega_{2+} ; x_{j}\right), & \text { otherwise, }\end{cases}
$$

where $\mathfrak{N}$ is the set of all the nodes of the mesh and $u_{n}\left(\Omega_{2-}, \Omega_{2+} ; x_{j}\right)$ is the linear interpolation of $u_{n}$ (see (2.9)) along the direction $\mathbf{n}(\beta)$.

To compute $E_{2 n}\left(\hat{l}_{20}, 1\right)$ and $l_{2 n}\left(\hat{l}_{20}, 1\right)$, the mesh transformation method [15] is applied to the problem (2.11), and the discrete minimization problem thus produced is solved by the conjugate gradient method. The idea of using the mesh transformation method is to let the mesh distribution to be involved into the minimization procedure so that the bending needle structure can be better resolved by the mesh lines on a relatively coarse mesh. The numerical results of $E_{2 n}\left(\hat{l}_{20}, 1\right)$ and $l_{2 n}\left(\hat{l}_{20}, 1\right)$ at some sample characteristic needle lengths are shown in table 1. Using the quadratic and cubic polynomials as the approximating functions for $E_{2 n}\left(\hat{l}_{20}, 1\right)$ and $l_{2 n}\left(\hat{l}_{20}, 1\right)$ respectively, by applying the least square method, we have

$$
\begin{gathered}
E_{2 n}\left(\hat{l}_{20}, 1\right) \approx a_{0}+a_{1} x+a_{2} x^{2}, \\
l_{2 n}\left(\hat{l}_{20}, 1\right) \approx b_{0}+b_{1} x+b_{2} x^{2}+b_{3} x^{3}
\end{gathered}
$$

with $a_{0}=3.489 \times 10^{-3}, a_{1}=-2.993 \times 10^{-4}, a_{2}=7.508 \times 10^{-6}, b_{0}=-69.58$, $b_{1}=17.76, b_{2}=-1, b_{3}=1.929 \times 10^{-2}$, and the corresponding numerical results are shown in Figure 5, where the numerical data in table 1 are marked by the $* \mathrm{~s}$ and the approximate polynomials (3.3) and (3.4) are shown in solid lines.

TABLE 1. Numerical results of $E_{n}, l_{n}$ for various $\hat{l}_{20}$

\begin{tabular}{|c|c|c|c|c|c|c|c|c|}
\hline$\hat{l}_{20}$ & 7.5 & 10 & 12.5 & 15 & 17.5 & 20 & 22.5 & 25 \\
\hline$E_{2 n}\left(\hat{l}_{20}, 1\right)\left(\times 10^{-3}\right)$ & 1.773 & 1.101 & 0.889 & 0.704 & 0.597 & 0.550 & 0.561 & 0.662 \\
\hline$l_{2 n}\left(\hat{l}_{20}, 1\right)$ & 15.23 & 27.53 & 34.40 & 37.57 & 36.19 & 40.22 & 45.18 & 50.13 \\
\hline
\end{tabular}



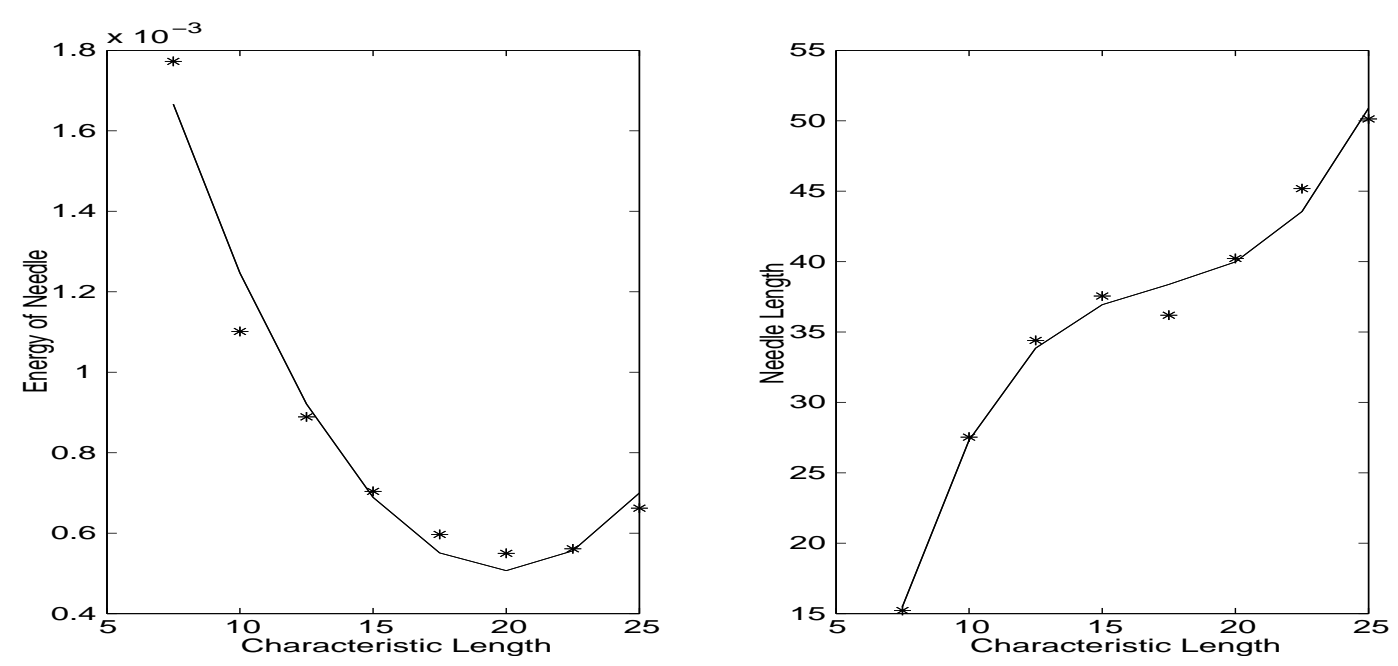

Figure 5. The numerical results of $E_{2 n}\left(\hat{l}_{20}, 1\right)$ and $l_{2 n}\left(\hat{l}_{20}, 1\right)$ for $\lambda=0.5$, and $\mu=1$.

In Figure 6 and Figure 7, we show two typical profiles of the needle structure on the correspondingly transformed $16 \times 16$ meshes for the characteristic needle lengths $\hat{l}_{20}=12.5,17.5$ respectively. Figure 8 shows the periodic structure of the laminated needles for $\hat{l}_{20}=17.5$.

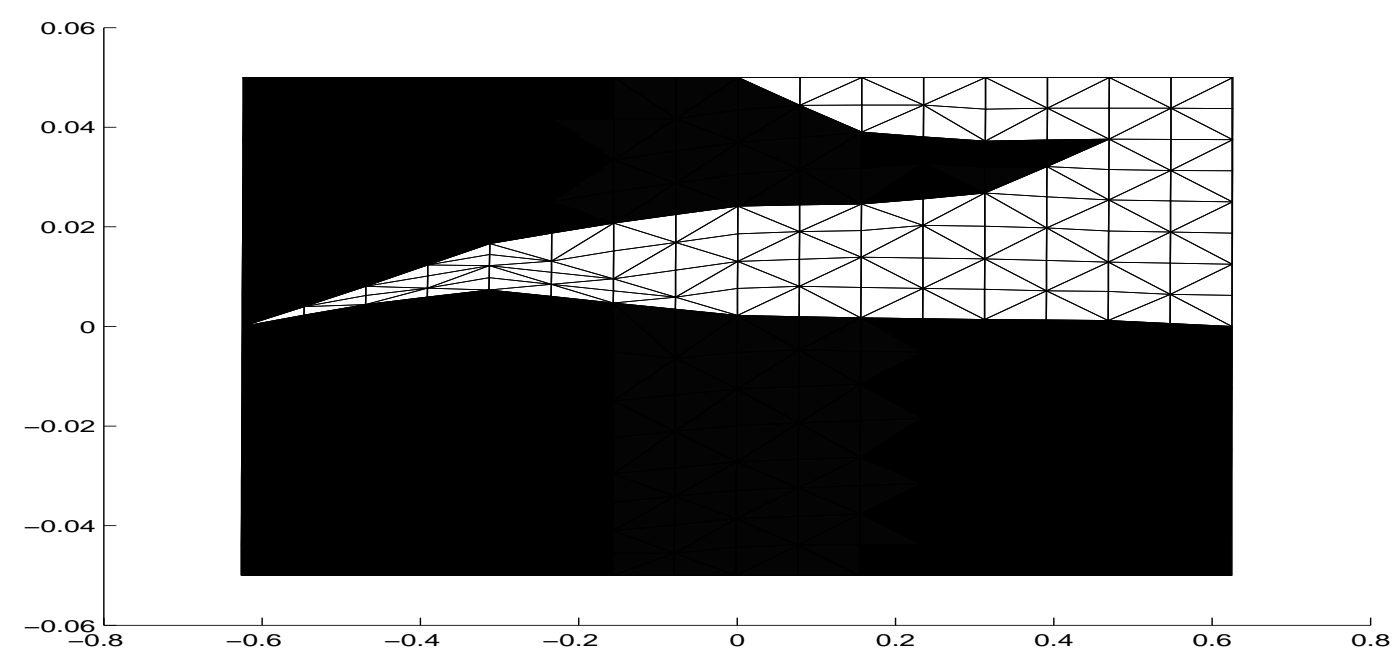

FigurE 6. Needle's profile for $\lambda=0.5, \mu=1$ and $\hat{l}_{20}=12.5$.

Similarly, We can establish the approximate function of $\tilde{E}_{b}(1, \hat{v})$. From the numerical results of $\tilde{E}_{b}(1, \hat{v})$ shown in Table 2 , we see that $\tilde{E}_{b}(1, \hat{v})$ is very large 


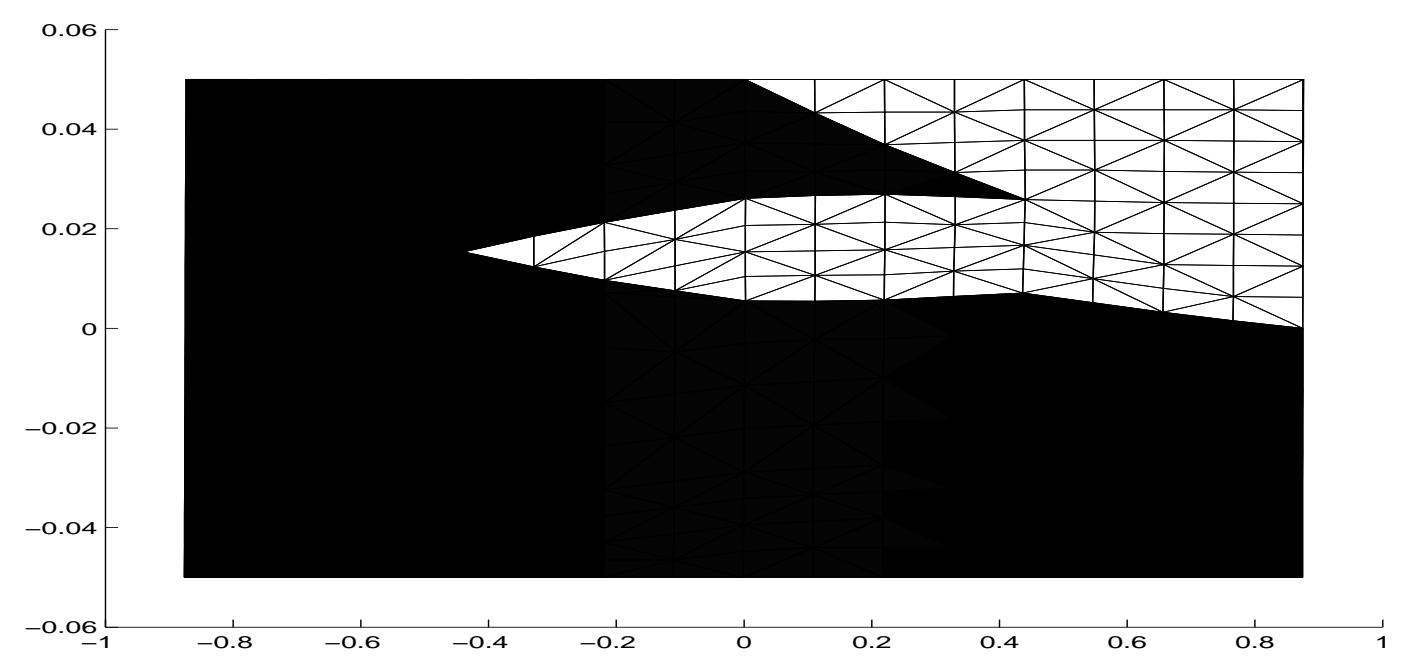

FiguRE 7. Needle's profile for $\lambda=0.5, \mu=1$ and $\hat{l}_{20}=17.5$.

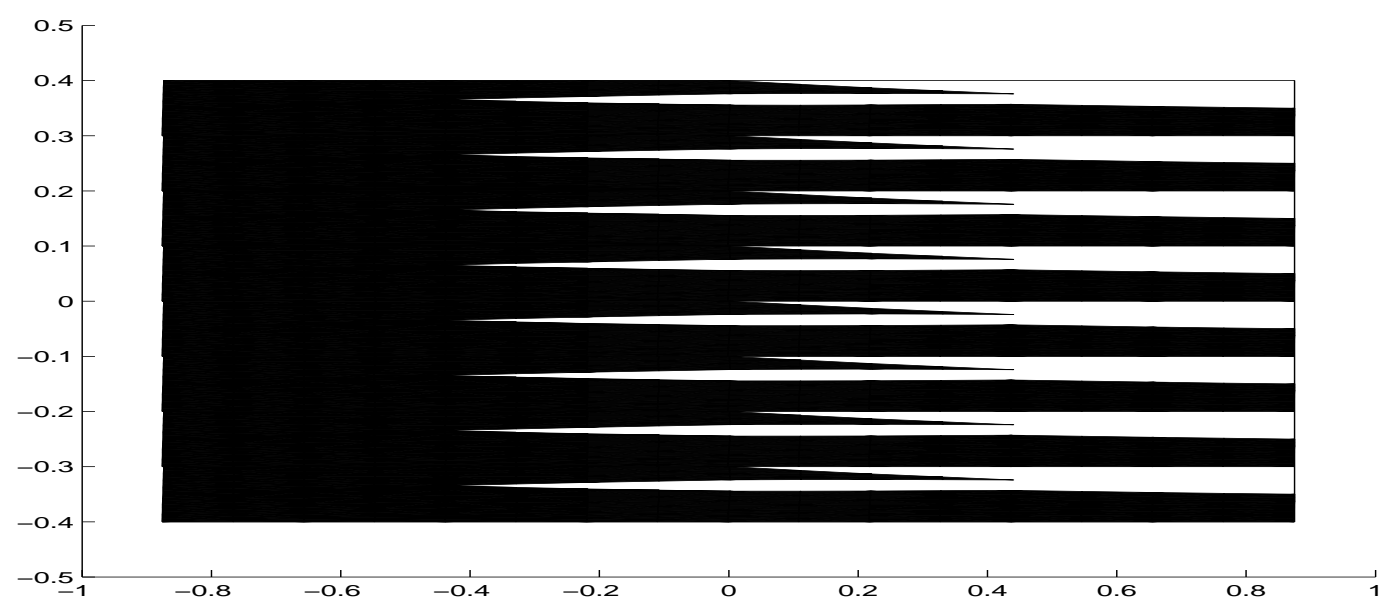

Figure 8. Laminated needles for $\lambda=0.5, \mu=1$ and $\hat{l}_{20}=17.5$.

when $\hat{v}$ is sufficiently small and is almost a linear function for $\hat{v} \in[0.4,1]$ and grows increasingly faster when $\hat{v}>1$. The approximate functions to $\tilde{E}_{b}(1, \hat{v})$ can be constructed in the three intervals. In Figure 9, where the data in Table 2 are marked by $*$ s, the numerical result of the approximate function to $E_{b}$ on the interval $[0.3,0.8]$ is shown, which is a linear function

$$
\tilde{E}_{b}(1, \hat{v}) \approx c_{0}+c_{1} \hat{v}
$$


for $\hat{v} \in[0.4,0.8]$ with $c_{0}=2.59 \times 10^{-11}, c_{1}=4.02 \times 10^{-4}$. Using the approximate functions given in (3.3), (3.4) and (3.5), for any given $\omega$, we can easily find, by solving the problem (2.29) with a 1-dimensional search, the approximate minimizing characteristic boundary layer width $\hat{\nu}(\omega)$, which is always found to be in the interval $(0.4,0.8)$ in our numerical experiments.

TABLE 2. Numerical results of $\tilde{E}_{b}(1, \hat{v})$ at various $\hat{v}$.

\begin{tabular}{|c|c|c|c|c|c|}
\hline$\hat{v}$ & 0.2 & 0.4 & 0.6 & 0.8 & 1.0 \\
\hline$\tilde{E}_{b}(1, \hat{v})$ & 2.57 & $1.56 \times 10^{-4}$ & $2.41 \times 10^{-4}$ & $3.22 \times 10^{-4}$ & $4.02 \times 10^{-4}$ \\
\hline$\hat{v}$ & 2.0 & 3.0 & 4.0 & 5.0 & 6.0 \\
\hline$\tilde{E}_{b}(1, \hat{v})$ & $8.85 \times 10^{-4}$ & $1.40 \times 10^{-3}$ & $5.03 \times 10^{-3}$ & $8.18 \times 10^{-2}$ & $1.19 \times 10^{-1}$ \\
\hline
\end{tabular}

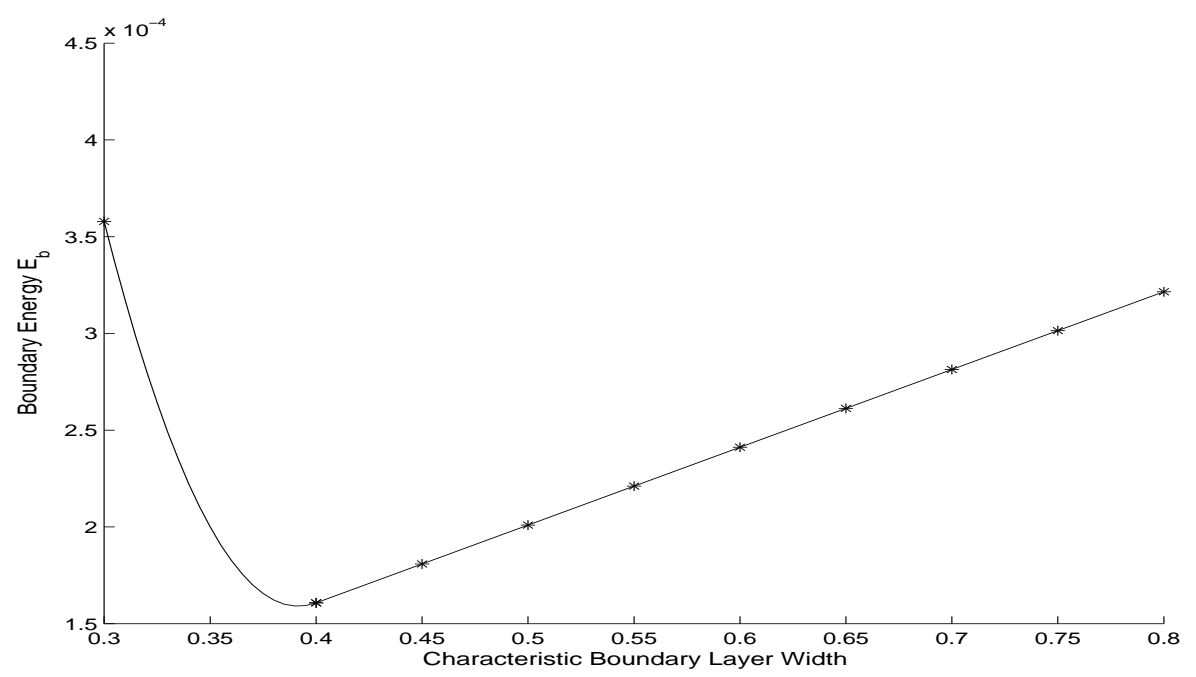

FiguRE 9. The numerical approximation of $\tilde{E}_{b}(1, \hat{v})$.

With the above preparation, we are now able to compute, by applying a 1dimensional search, the numerical solutions to the problem (2.30) and thus the length scales for the second order laminated microstructures. In Table 3, the numerical results on the twin width $\omega$ of the first order laminate and the twin width $\omega_{2}$ of the second order laminate (Martensite-2) and the average energy density $\bar{E}_{T}$ of the corresponding second order laminated microstructure, for the surface energy density $e_{s}=10^{-8}$ and various sample lengths $l$, are shown. Furthermore, 
TABLE 3. The numerical results on $\omega, \omega_{2}$ and $\bar{E}_{T}$ for various $l$, in the case of $e_{s}=10^{-8}$.

\begin{tabular}{|c|c|c|c|c|c|c|c|c|}
\hline$l$ & 100 & 200 & 300 & 400 & 500 & 600 & 700 & 800 \\
\hline$\omega$ & 2.70 & 4.30 & 5.64 & 6.83 & 7.93 & 8.95 & 9.92 & 10.84 \\
\hline$\omega_{2}\left(\times 10^{-4}\right)$ & 0.721 & 0.909 & 1.004 & 1.146 & 1.234 & 1.312 & 1.381 & 1.444 \\
\hline $\bar{E}_{T}\left(\times 10^{-6}\right)$ & 10.93 & 8.66 & 7.56 & 6.86 & 6.37 & 5.99 & 5.69 & 5.44 \\
\hline
\end{tabular}

our numerical experiments show that, for different $l$ and $e_{s}$, the characteristic needle length $\hat{l}_{20}$ and the characteristic boundary layer width $\hat{v}$ vary only slightly in a neighborhood of 18.8 and 0.5 respectively, and $E_{2 n}\left(\hat{l}_{20}, 1\right)$ is almost a constant. Thus, by (2.18) and (2.27), we see that the twin width $\omega_{2}$ of the Martensite- 2 laminate is approximately a linear function of $\left(\omega e_{s}\right)^{\frac{1}{2}}$, that is

$$
\omega_{2} \approx C_{1}\left(\omega e_{s}\right)^{\frac{1}{2}}
$$

where $C_{1}$ is approximately a constant which in our case is about 43.8 .

Recalling that the Martensite- 1 is in this case a pure martensite variant and the average total energy density of the second order laminated microstructure is of the form

$$
\begin{gathered}
\bar{E}_{T}(l, \omega, \hat{\nu}(\omega))=\Phi_{\theta}+\left[2\left(2 l_{2}(\omega)\left(E_{2 n}\left(\hat{l}_{20}(\omega), 1\right)-\Phi_{\theta} \hat{l}_{20}(\omega)\right) e_{s}\right)^{\frac{1}{2}}+\right. \\
\left.\left(l_{2 n}\left(\hat{l}_{20}(\omega), 1\right)-2 \hat{l}_{20}(\omega)\right) e_{s}\right] \frac{1}{\omega}+2\left[\tilde{E}_{b}(1, \hat{v}(\omega)) \omega-E_{2}\left(l_{2}(\omega), \hat{l}_{20}(\omega)\right) \hat{v}(\omega)\right] \frac{1}{l}
\end{gathered}
$$

where $l_{2}(\omega)$ is given by $(2.27), \hat{l}_{20}(\omega) \approx 18.8$ and $\hat{v}(\omega) \approx 0.5$ are the corresponding minimizing characteristic needle length and boundary layer width, we have the following reduced formula

$$
\bar{E}_{T}(l, \omega, \hat{\nu}(\omega)) \approx \Phi_{\theta}+C_{2}\left(\frac{e_{s}}{\omega}\right)^{\frac{1}{2}}+C_{3} \frac{\omega}{l} .
$$

Thus, by minimizing the right side of (3.7), we obtain the approximate scaling law for $\omega$ and the minimum average energy density $\bar{E}_{T}$ :

$$
\omega \approx C_{4}\left(l^{2} e_{s}\right)^{\frac{1}{3}}, \quad \bar{E}_{T} \approx C_{5}\left(l^{-1} e_{s}\right)^{\frac{1}{3}} .
$$


In fact, our numerical experiments show that $C_{4}$ and $C_{5}$ can be approximated in high precision with the following functions

$$
C_{4} \approx C_{40}+C_{41} \exp \left(C_{42} e_{s}\right), \quad C_{5} \approx C_{50}+C_{51} \exp \left(C_{52} e_{s}\right)
$$

with $C_{40}=58.205, C_{41}=82.967, C_{42}=-0.75, C_{50}=2.3461 \times 10^{-2}, C_{51}=$ $1.1572 \times 10^{-2}, C_{52}=-0.75$ in our case, which tend to constants exponentially as $e_{s}$ goes to zero. In Table 4 , some numerical results on $C_{4}$ and $C_{5}$ are shown.

TABle 4 . The numerical results for $C_{4}, C_{5}$ for various $e_{s}$

\begin{tabular}{|c|c|c|c|c|c|c|c|c|}
\hline$\sigma_{s}\left(\times 10^{-8}\right)$ & 1 & 2 & 3 & 4 & 5 & 6 & 7 & 8 \\
\hline$C_{4}$ & 58.410 & 58.463 & 58.500 & 58.530 & 58.554 & 58.576 & 58.596 & 58.613 \\
\hline$C_{5}\left(\times 10^{-2}\right)$ & 2.3490 & 2.3497 & 2.3502 & 2.3506 & 2.3510 & 2.3512 & 2.3515 & 2.3518 \\
\hline
\end{tabular}

The numerical results on the relations between the twin width $\omega$, the average energy density $\bar{E}_{T}$ and the sample length $l$ for the surface energy density $\left\{e_{s}^{i}\right\}_{i=1}^{10}=$ $i \times 10^{-8}$ are shown in Figure 10 and Figure 11 .

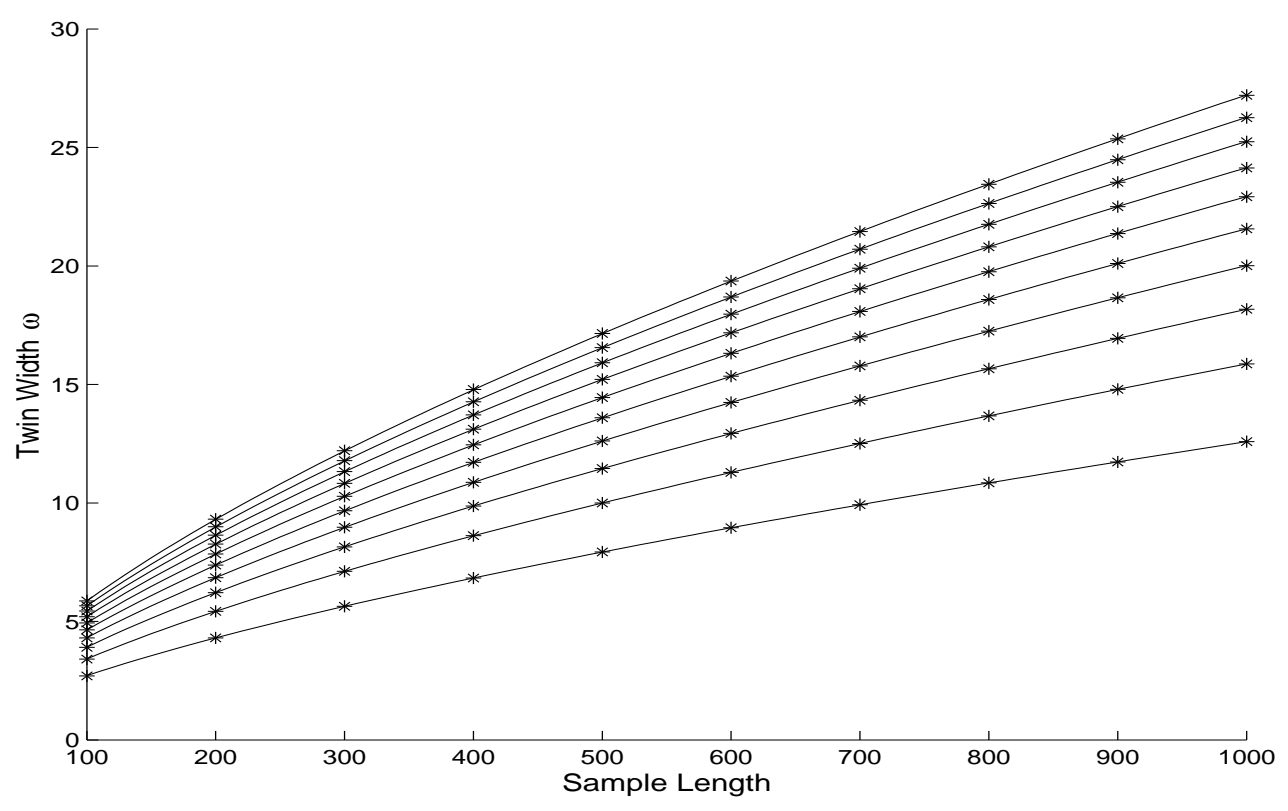

Figure 10. The relation between $\omega$ and $l$ for various $e_{s}$. 


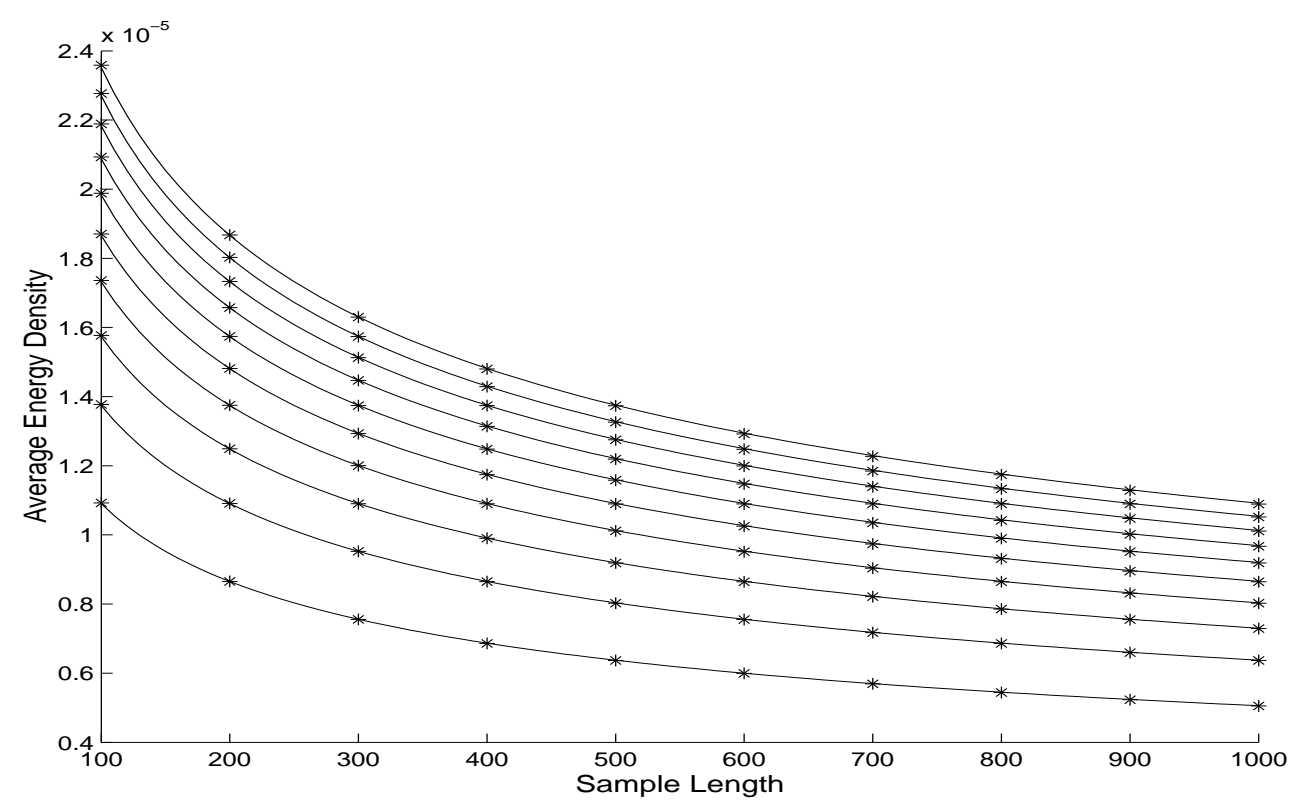

FiguRE 11. The relation between $\bar{E}_{T}$ and $l$ for various $e_{s}$.

As a conclusion, by (3.6), (3.8) and (3.9), we have the following cubic rule for the scaling law

$$
\omega \approx C_{4}\left(l^{2} e_{s}\right)^{\frac{1}{3}}, \quad \omega_{2}=C_{\omega_{2}}\left(l e_{s}^{2}\right)^{\frac{1}{3}}
$$

where

$$
C_{\omega_{2}}=C_{1} C_{4}^{\frac{1}{2}} \approx C_{1}\left(C_{40}+C_{41} \exp \left(C_{42} e_{s}\right)\right)^{\frac{1}{2}},
$$

which tend to 334.16 , as $e_{s} \rightarrow 0$ in our case.

\section{REFERENCES}

[1] J. M. Ball and R. D. James, Fine phase mixtures as minimizers of energy. Arch. Rat. Mech. Anal., 100(1987)1, 13-52.

[2] J.M. Ball, A version of the fundamental theorem for Young measures, in Partial Differential Equations and Continuum Models of Phase Transitions, ( M. Rascle, D. Serre and M. Slemorod, eds ), Lecture Notes in Physics, 344, 207-215, 1989.

[3] J. M. Ball and R. D. James, Proposed experimental test of a theory of fine microstructure and the two-well problem. Phil. Trans. R. Soc. London 338A(1992), 389-450.

[4] M. Chipot, Elements of Nonlinear Analysis, Boston: Birkhuser Verlag, 2000.

[5] S. Conti, Branched microstructures: scaling and asymptotic self-similarity. Comm. Pure Appl. Math., 53(2000), 1448-1474. 
[6] B. Dacorogna, Direct Methods in the Calculus of Variations, Applied Mathematical Sciences, 78, Springer-Verlag, Berlin, 1989.

[7] R. Kohn, Relaxation of a double-well energy, Continuum Mechanics and ThermoDynamics, 3(1992), 193-236.

[8] R.V. Kohn and Stefan Müller, Branching of twins near an austenite-twinned-martensite interface. Phil. Mag., A 66(1992), 697-715.

[9] R.V. Kohn and Stefan Müller, Surface energy and microstructure in coherent phase transitions. Comm. Pure Appl. Math., 47(1994), 405-435.

[10] B. Li and M. Luskin, Theory and computation for the microstructure near the interface between twinned layers and a pure variant of martensite, Materials Science and Engineering, A 273(1999), 237-240.

[11] Z.-P. Li, Rotational transformation method and some numerical techniques for the computation of microstructures. Math. Models Meth. Appl. Sci., 8(1998), 985-1002.

[12] Z.-P. Li, Finite order rank-one convex envelopes and computation of microstructures with laminates in laminates. BIT, 40(4)(2000), pp.745-761.

[13] Z.-P. Li, A periodic relaxation method for computing microstructures. Appl. Numer. Math., 32(2000), 291-303.

[14] Z.-P. Li, Computations of needle-like microstructures, Appl. Numer. Math., 39(2001), $1-15$.

[15] Z.-P. Li, A mesh transformation method for computing microstructures. Numer. Math., 89(2001), pp. 511-533.

[16] Z.-P. Li, A numerical study on the scale of laminated microstructure with surface energy. Materials Science and Engineering, A 343(2003), pp. 182-193.

[17] Z.-P. Li, Numerical justification of branched laminated microstructure with surface energy. SIAM J. Sci. Compt., 24(2003), pp. 1054-1075.

[18] M. Luskin, Computational modeling of microstructure, ICM2002, Vol.3, 707-716.

[19] M. Luskin, On the computation of crystalline microstructure. Acta Numerica, 5(1996), 191-257.

[20] M. Luskin, The computation of martensitic microstructure with piecewise laminates, J. Sci. Comput., 19(2003), 293-308.

[21] P. Bêlik and M. Luskin, A total-variation surface energy model for thin films of martrensitic crystals, Interfaces and Free Boundariers, 4(2002), 71-88.

[22] X.-W. Yu, Z.-P. Li and L.-A. Ying, Singular perturbation of a class of non-convex functionals, Nonlinear Analysis 52(2003), 1129-1152. 